This is an Open Access article, distributed under the terms of the Creative Commons Attribution licence (http://creativecommons.org/licenses/by/4.0/), which permits unrestricted re-use, distribution, and reproduction in any medium, provided the original work is properly cited.

\title{
Proton acceleration in a laser-induced relativistic electron vortex
}

\author{
L. Q. Yi ${ }^{\oplus 1} \dagger$, I. Pusztai ${ }^{\oplus 1}$, A. Pukhov ${ }^{2}$, B. F. Shen ${ }^{3}$ and T. Fülöp ${ }^{\oplus 1}$ \\ ${ }^{1}$ Department of Physics, Chalmers University of Technology, SE-41296 Göteborg, Sweden \\ ${ }^{2}$ Institut für Theoretische Physik I, Heinrich-Heine-Universität Düsseldorf, Düsseldorf, 40225, Germany \\ ${ }^{3}$ Department of Physics, Shanghai Normal University, Shanghai, 200234, China
}

(Received 12 April 2019; revised 17 June 2019; accepted 18 June 2019)

We show that when a solid plasma foil with a density gradient on the front surface is irradiated by an intense laser pulse at a grazing angle, $\sim 80^{\circ}$, a relativistic electron vortex is excited in the near-critical-density layer after the laser pulse depletion. The vortex structure and dynamics are studied using particle-in-cell simulations. Due to the asymmetry introduced by non-uniform background density, the vortex drifts at a constant velocity, typically $0.2-0.3$ times the speed of light. The strong magnetic field inside the vortex leads to significant charge separation; in the corresponding electric field initially stationary protons can be captured and accelerated to twice the velocity of the vortex (100-200 MeV). A representative scenario - with laser intensity of $10^{21} \mathrm{~W} \mathrm{~cm} \mathrm{~cm}^{-2}$ - is discussed: two-dimensional simulations suggest that a quasi-monoenergetic proton beam can be obtained with a mean energy $140 \mathrm{MeV}$ and an energy spread of $\sim 10 \%$. We derive an analytical estimate for the vortex velocity in terms of laser and plasma parameters, demonstrating that the maximum proton energy can be controlled by the incidence angle of the laser and the plasma density gradient.

Key words: plasma applications, plasma dynamics, plasma simulation

\section{Introduction}

Acceleration of protons by intense lasers has attracted extensive interest due to the potential significance in several branches of science, technology and medicine (Daido, Nishiuchi \& Pirozhkov 2012). The most experimentally robust mechanism for laserdriven proton acceleration is target-normal sheath acceleration (TNSA) (Pukhov 2001; Wilks et al. 2001; Roth et al. 2002; Mora 2003; Fuchs et al. 2006), where protons are accelerated by the electrostatic sheath field arising due to the expansion of laser-heated electrons. It usually leads to a broad proton energy spectrum due to lack of control of the heating process (Daido et al. 2012), and the maximum achieved proton energy of $94 \mathrm{MeV}$ (Higginson et al. 2018), is still not sufficiently high for many of the foreseen applications; e.g. hadron therapy requires 100-200 MeV monoenergetic proton beams

† Email address for correspondence: longqing@chalmers.se 


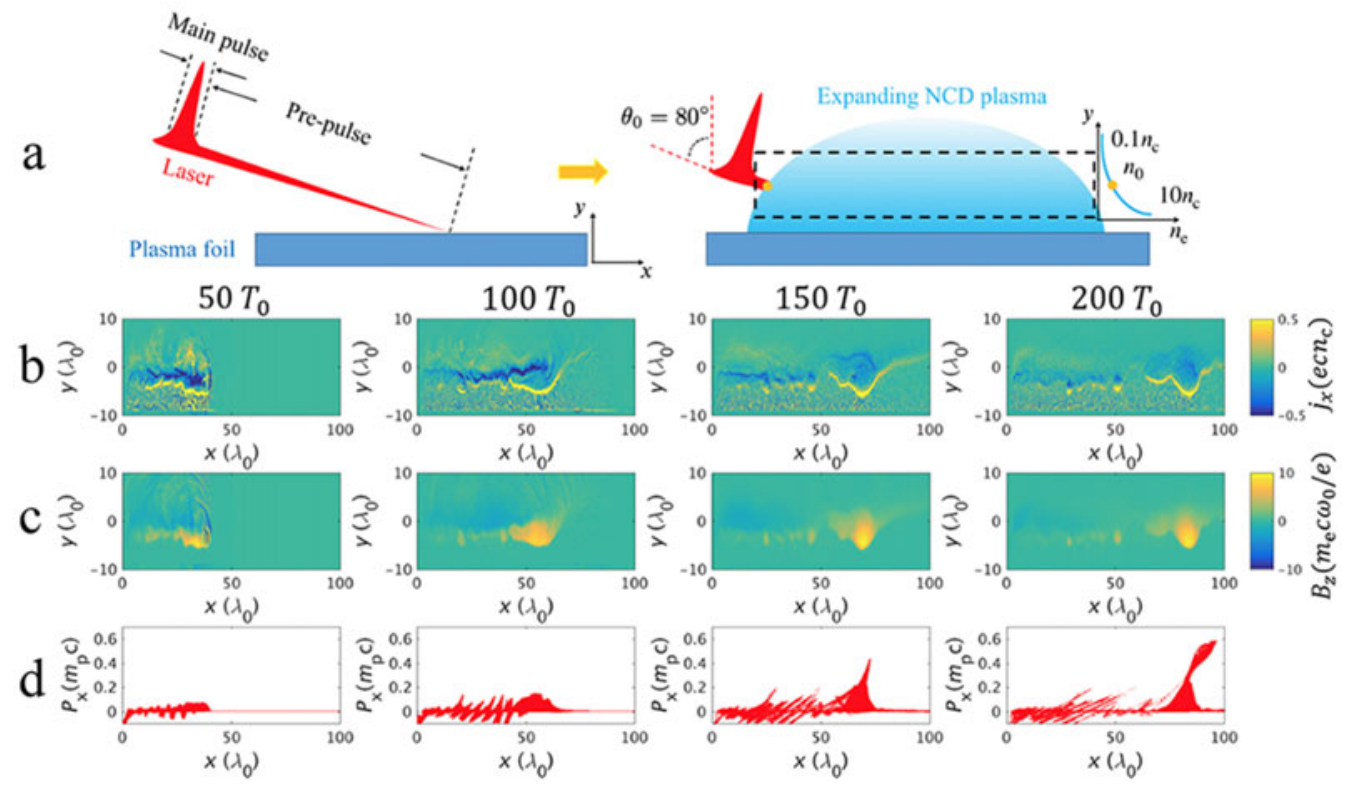

FIGURE 1. Schematic of the proposed proton acceleration set-up. (a) A laser pulse grazing incident on a plasma foil (left). After heating by the laser pre-pulse, an expanding plasma region is formed on the surface (cyan shaded region), in which the main pulse interacts with the NCD layer (right). The black dashed box represents the simulation area, the orange dot marks the main pulse incident point, where the density is $n_{0} \approx 0.4 n_{\mathrm{c}}$. Snapshots of $(b)$ the current density parallel to the target surface $j_{x},(c)$ the out-of-plane magnetic field $B_{z}$ and $(d)$ the proton phase space for the $x$-direction, are shown at $t=$ $50 T_{0}, 100 T_{0}, 150 T_{0}$ and $200 T_{0}$, respectively.

(Bulanov et al. 2008). In other proposed schemes, the acceleration usually involves a slow-moving acceleration structure (i.e. such that protons can keep up with it) that can be driven by the piston action of the laser, radiation pressure or a shock wave that is detached from the laser (Macchi, Borghesi \& Passoni 2013; Borghesi \& Macchi 2016; Schreiber, Bolton \& Parodi 2016; Macchi 2017). The protons then stay in phase with this structure and get energised over a longer period of time, which typically leads to higher cutoff energies. However, in most cases the acceleration is strongly limited by the instabilities on the interface where the light pressure is balanced by the plasma fields (Pegoraro \& Bulanov 2007; Palmer et al. 2012; Khudik et al. 2014).

In this paper, we describe a new proton acceleration mechanism that relies on a laser-induced relativistic electron vortex $(\mathrm{EV})$ in a near-critical-density (NCD) plasma with a density gradient normal to the target surface (see figure $1 a$ ). The EVs are not susceptible to the instabilities arising at the laser-plasma interface, as they form after the laser depletion. Here the EVs are micro-scale, long lived, coherent magnetic flux ropes with a unipolar magnetic structure in two dimensions, and share similarities with a particular class of stable solutions to the electron magnetohydrodynamic equations (Nycander \& Pavlenko 1991; Gordeev \& Levchenko 1998; Yadav, Das \& Kaw 2008; Richardson et al. 2013; Angus et al. 2014). Unlike the classic TNSA scheme, the acceleration mechanism described here is able to produce a quasi-monoenergetic proton beam, and the drift velocity of the EV - and thus the beam energy - can be 
tuned more easily compared to the collisionless shock acceleration (Silva et al. 2004; Fiuza et al. 2012; Haberberger et al. 2012; Pak et al. 2018). In addition to the application for proton acceleration, which is our main focus here, EVs have numerous potential applications. The strong coherent magnetic fields can be utilised in studying reconnection (Priest, Hornig \& Pontin 2003; Yamada, Kulsrud \& Ji 2010; Yi et al. 2018), or the evolution of magnetic helicity in a kinetic system (Rust \& Kumar 1996; Wiegelmann \& B"uchner 2001), thereby representing a fundamentally interesting setting to the wider plasma physics community.

Previous particle-in-cell (PIC) simulations have shown vortices in connection with a high intensity laser propagating through an underdense plasma (Bulanov et al. 1996; Naumova et al. 2001), but their potential for proton acceleration was not considered. In this work, we show that with a high-intensity laser at grazing incidence on a solid plasma foil, a strong relativistic EV can be excited in a NCD plasma layer after the laser depletion. Although the physics of laser pulse propagation in NCD plasmas has been widely studied (Pukhov \& Meyer-ter Vehn 1996; Nakamura \& Mima 2008; Bulanov et al. 2010; Liu et al. 2013; Bin et al. 2015; Liu et al. 2016; Hilz et al. 2018; Ma et al. 2019), the current study focuses a new regime, where the plasma density inhomogeneity length scale is comparable to the vortex size in the direction normal to the surface of the target $(y)$, while in the tangential directions, the density length scale is much larger. Such density profile can be created with the pre-pulse of the laser evaporating the front surface of a solid plasma foil. Alternatively, when a sufficiently high contrast is achieved (Thaury et al. 2007), the pre-plasma can be created by another laser with very large spot size irradiating the target prior to the main pulse (Gauthier et al. 2014). The acceleration mechanism considered here is different from the previously studied magnetic vortex acceleration (MVA) (Bulanov \& Esirkepov 2007; Bulanov et al. 2010; Nakamura et al. 2010; Lemos et al. 2012), where a bipolar magnetic vortex is generated in a uniform NCD target (Nakamura \& Mima 2008) and acceleration only happens when it reaches the rear side of the target. In our work, the EV, or unipolar magnetic vortex, serves as a stable slow-moving structure in which protons can be captured, and gain considerable kinetic energy from the charge separation field associated with the EV. Similarly to collisionless shock acceleration, protons initially at rest can be reflected to twice the EV drift velocity (Macchi et al. 2013), and a narrow energy spectrum can be obtained in the direction of the vortex propagation.

\section{EV formation and the proton acceleration}

Figure 1 shows the simulation set-up. A linearly (p-)polarised laser beam with normalised amplitude $a_{0}=30$ (intensity $I=1.2 \times 10^{21} \mathrm{~W} \mathrm{~cm}{ }^{-2}$ ), is incident at a grazing angle of $80^{\circ}$ on a plasma foil. Here $a_{0}=e E_{0} / m_{\mathrm{e}} c \omega_{0}, E_{0}, \omega_{0}$ and $\lambda_{0}=1 \mu \mathrm{m}$ are the peak electric field, frequency and wavelength of the laser pulse, while $c, e$ and $m_{\mathrm{e}}$ denote the speed of light in vacuum, the elementary charge and electron mass, respectively. The focal spot and the full width at half maximum (FWHM) duration of the main laser pulse are $w_{0}=3 \lambda_{0}$ and $5.4 T_{0}=18 \mathrm{fs}$, respectively, where $T_{0}$ is the laser period. The main laser pulse propagates to the right with a grazing angle with respect to the target surface. It first penetrates the underdense plasma region until it reaches the simulation box (marked by the black-dashed line in figure 1, there is a $2 \mu \mathrm{m}$ vacuum gap between the plasma and the left boundary where laser is injected), the density at the incident point is $n_{0} \approx 0.4 n_{c}$ (in reality this is the position where the density becomes strongly inhomogeneous). The density profile 
within the simulation box is initialised with a density decreasing exponentially in the $y$ direction as $n_{\mathrm{e}}(y)=100 n_{\mathrm{c}} \exp \left[-\left(y-y_{\mathrm{t}}\right) / \sigma\right]$, where $n_{\mathrm{c}}=m_{\mathrm{e}} \omega_{0}^{2} / 4 \pi e^{2}$ is the critical density, $y_{\mathrm{t}}=-16.9 \lambda_{0}$ is the target position and $\sigma=3 \lambda_{0}$ is the scale length. The density profiles in the tangential directions are assumed to be uniform since the scale lengths in those directions are much larger than $\sigma$. Note that it is possible to achieve such density profile experimentally, as reported by Gauthier et al. (2014). The proton density is $n_{\mathrm{p}}(y)=n_{\mathrm{e}}(y) / 55$. The proton species is assumed to consist only a small fraction of the total positive charge, the majority of ions are considered to be immobile in this simulation. The effect of heavy ion motion will be addressed in the discussion section. The PIC simulations are performed with EPOCH (Arber et al. 2015). The simulation window $x \times y=200 \lambda_{0} \times 20 \lambda_{0}$ is sampled by $4000 \times 400$ cells with 10 macro electrons and 20 macro protons in each cell.

Figure $1(b, c)$ shows the evolution of the current density $\left(j_{x}\right)$ and the out-of-plane magnetic field $\left(B_{z}\right)$ during the interaction. The first column $\left(t=50 T_{0}\right)$ shows the laser channelling in the NCD plasma, and the associated strong currents inside the channel due to the laser-plasma interaction. In the NCD plasma, the laser effectively transfers all its energy to the electrons within a distance of a few tens of microns (Sylla et al. 2013). In the second column $\left(t=100 T_{0}\right)$, the laser energy is completely depleted and the electron dynamics is dominated by self-generated fields. The electrons gyrate in the self-generated magnetic fields, forming a stable vortex structure. The two columns on the right $\left(t=150 T_{0}\right.$ and $200 T_{0}$ ) show that the EV continues to propagate along $x$ with a drift velocity $\sim 0.28 c$. In the meantime, the background protons are accelerated in the $x$-direction by the EV as shown in figure $1(d)$.

The acceleration of protons is due to the charge separation caused by the magnetic pressure in the EV. As shown by figure $2(a, b)$, the electron density inside the EV is lower than the ambient value. A high-density return current layer forms on the lower boundary of the EV. Components of the electrostatic field induced by the charge separation are presented in figure $2(c, d)$.

As the EV drifts, the protons are accelerated in the leading half of the structure. Those protons that gain enough energy can keep up with the EV (they are captured), otherwise they lose energy due to the decelerating field in the second half of the EV. The captured protons can be continuously accelerated until they overrun the vortex with approximately twice its drift velocity.

The electric field in the direction normal to the target surface $\left(E_{y}\right)$ is defocusing, therefore only a fraction of the captured protons can achieve the maximum energy gain, others are scattered out of the acceleration phase before they can do so. This makes the energy distribution depend strongly on the pitch angle as indicated by figure $2(e)$, where the slope of the dots shows the pitch angle $\theta_{\mathrm{p}}=P_{y} / P_{x}$ of the corresponding protons. The energy spread of the accelerated protons can then be adjusted by selecting different pitch angles (e.g. with a collimator): at large $\theta_{\mathrm{p}}$ a wide spectrum is obtained with a sudden cutoff, while protons at low $\theta_{\mathrm{p}}$ exhibit a quasi-monoenergetic feature. External focusing with various well-established approaches (Toncian et al. 2006; Albertazzi et al. 2015; Zhai et al. 2019) can be reliably employed in order to obtain a well-collimated beam for applications such as cancer therapy.

Figure $2(f)$ presents the energy spectrum of the protons within a $10^{\circ}$ opening angle in the forward direction. The proton beam has a mean energy $\sim 140 \mathrm{MeV}$ and an energy spread of approximately $10 \%$. In this quasi-monoenergetic beam the proton number per $1 \%$ energy range and solid angle increment $\Delta \Omega$ is estimated to 

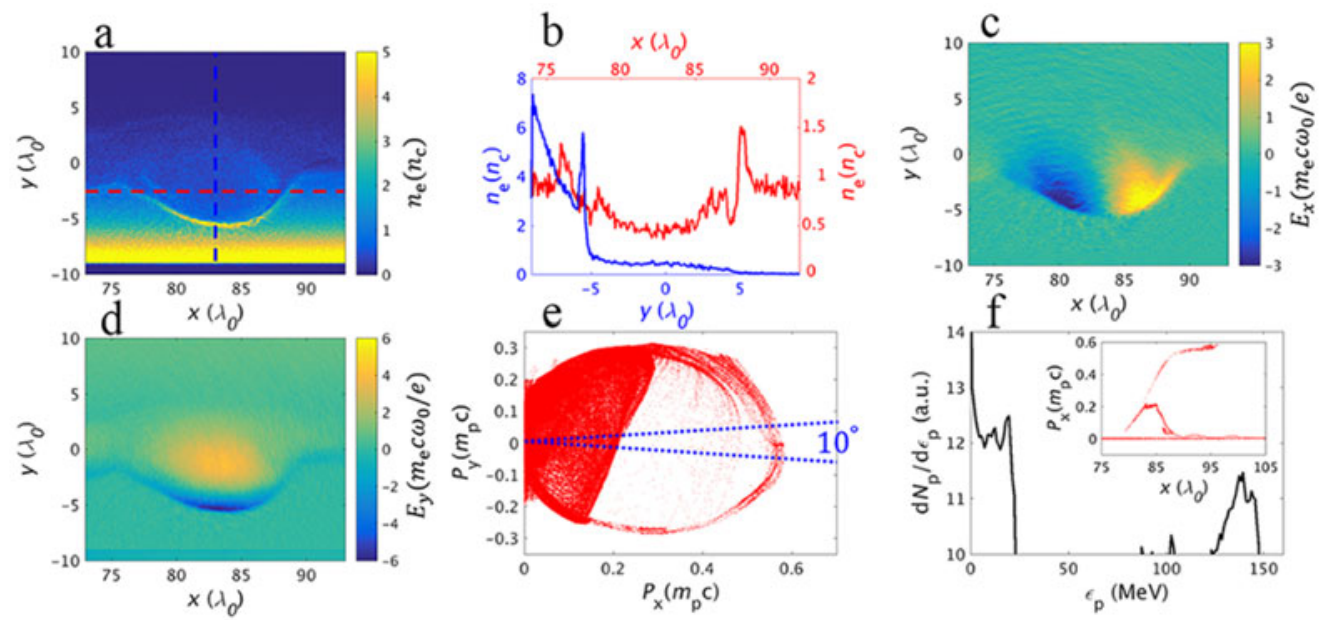

FIGURE 2. Features of the relativistic EV. (a) The electron density distribution, where (b) presents the one-dimensional cut of electron density along the horizontal (red) and vertical (blue) axes (marked as dashed in $a$ ). Electric field components in the directions $(c)$ parallel and $(d)$ normal to the target surface. (e) Proton population in momentum space $P_{x}-P_{y}$, and $(f)$ energy spectrum of protons within opening angle $10^{\circ}$ (between blue dashed lines in $e$ ). The inset in $(f)$ shows the phase space map $x-P_{x}$ of these protons. All quantities are shown at simulation time $t=200 T_{0}$.

be $\sim 10^{6} / \mathrm{msr} / 1 \% \epsilon_{\mathrm{p}}$ (assuming the proton beam to be axisymmetric with respect to the $x$ axis), which is comparable to contemporary laser-driven ion acceleration schemes at their cutoff energy (Schreiber et al. 2016), and this can be further improved by increasing the proton-to-heavy-ion ratio in the plasma.

In order to obtain a deeper insight into the electron dynamics, we track 2000 electrons throughout the simulation. These electrons are chosen to be located inside the $\mathrm{EV}$ at $t=150 T_{0}$, with half of them in the forward-going current distributed throughout the structure (hereafter laser-induced current) and the other half in the return current. Their positions at $t=150 T_{0}$ and $t=400 T_{0}$ are represented by blue and red circles in figure 3(a), respectively. Most of the electrons in the laser-induced current stay within the EV, but the electrons in the return current are lost when they reach the end of the EV. This can also be seen from the representative orbits shown by the black curves.

An individual electron drifts in the electric and magnetic fields self-generated by the vortex, where the drift velocity can be obtained by the sum of the $\boldsymbol{E} \times \boldsymbol{B}$ drift and grad- $B$ drift. (Note that only the electrons are magnetised, so the protons and ions are not subject to $\boldsymbol{E} \times \boldsymbol{B}$ drift in the time scale of interest)

$$
\frac{\boldsymbol{v}_{\mathrm{d}}}{c}=\frac{\boldsymbol{E} \times \boldsymbol{B}}{B^{2}}-\left(\gamma_{\mathrm{e}}-\frac{1}{\gamma_{\mathrm{e}}}\right) \frac{m_{\mathrm{e}} c^{2} \boldsymbol{B} \times \nabla B}{2 e B^{3}},
$$

where $\gamma_{\mathrm{e}}$ is the relativistic factor of electrons. By assuming $\gamma_{\mathrm{e}}=\gamma_{a} \equiv \sqrt{1+a_{0}^{2} / 2}$ in the laser-induced current and $\gamma_{\mathrm{e}} \sim 1.4$ in the relatively cold return current, the prediction of (2.1) matches the PIC simulation data as shown by figure 3(b). Moreover, it can be seen that the $\boldsymbol{E} \times \boldsymbol{B}$ drift is the dominating term in (2.1); the grad- $B$ drift only becomes 

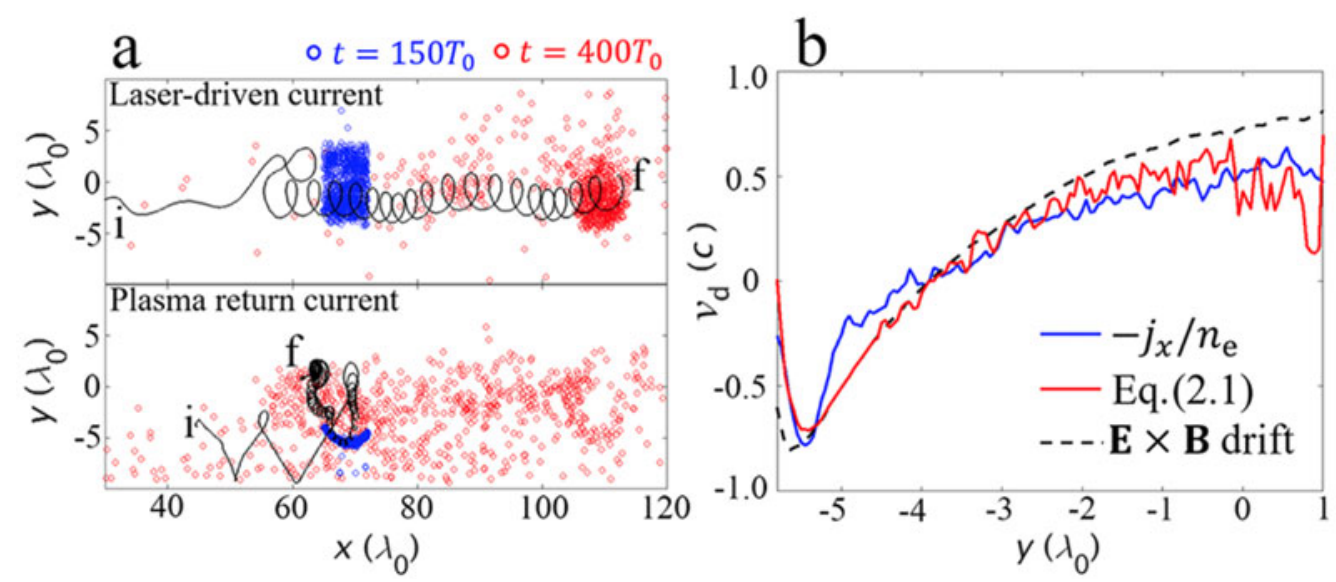

FIgURE 3. The dynamics of the trapped electrons in the EV. (a) The upper and lower plots show the positions of 1000 tracked electrons (each) in the laser-driven current and return current, selected at $t=150 T_{0}$. The red and blue circles show their position at $t=150 T_{0}$ and $t=400 T_{0}$, respectively. A representative electron trajectory in each case is plotted with the black curve, $\mathrm{i}$ and $\mathrm{f}$ mark the initial and final points of the trajectory. (b) A comparison of the average drift velocity of electrons (blue line) and the prediction from (2.1) (red line), the black dashed line shows the $\boldsymbol{E} \times \boldsymbol{B}$ drift velocity (first term in (2.1)). All quantities are taken from the simulation at $t=200 T_{0}$, along $x=83.5 \lambda_{0}$.

important in the upper boundary of the EV, where the gyro-radii of electrons are not negligible compared to the variation scale of magnetic fields.

\section{Model of EV velocity in strongly non-uniform plasmas}

Since the laser-induced electrons propagate with the EV (see figure 3(a) and supplementary movie, available at https://doi.org/10.1017/S0022377819000485), their average drift velocity must equal the EV propagation speed. Due to the approximate anti-symmetry of $E_{x}$, shown in figure 2 , the drifts in $y$ direction must average to zero and the EV moves in a direction consistent with $\boldsymbol{B} \times \nabla n$, as found in weakly inhomogeneous plasmas (Richardson et al. 2013; Angus et al. 2014).

We model the EV as two oppositely travelling electron streams: the electrons going backwards are in a thin layer with high density, and the ones going forward are in a larger region but with significantly lower density, as shown in figure 4(a). For simplicity, the electron and ion densities are taken to be uniform in the return current layer: $n_{\mathrm{e}}=n_{\mathrm{e} 1}$ and $n_{\mathrm{i}}=n_{\mathrm{i} 1}$, and the $y$ coordinate is set to be zero at the bottom of the EV. The interface between the two oppositely travelling currents is at $y=y_{1}$ and the upper boundary of the $\mathrm{EV}$ is at $y=y_{2}$ (where the gyro-radii of the electrons become comparable to the size of EV). As the proton density is negligible, the positive charge density is $\operatorname{Zen}_{\mathrm{i} 1} \exp \left[-\left(y-y_{1}\right) / \sigma\right]$, where $Z$ is the charge number and $n_{\mathrm{i} 1}$ is the immobile ion density at $y=y_{1}$. Furthermore, we assume the shape of $\mathrm{EV}$ to be elongated in the $x$ direction (which is increasingly well satisfied for high $\left.a_{0}\right)$, effectively reducing the problem to one dimension $(\partial / \partial x \ll \partial / \partial y)$. The relevant electric and magnetic fields at $y_{1}$ are $E_{y}=E_{1}, B_{z}=B_{1}$, and $E_{2}, B_{2}$ at $y_{2}$, respectively, where $\left|B_{2}\right| \ll\left|B_{1}\right|$ and $\left|E_{2}\right| \ll\left|E_{1}\right|$.

The electric and magnetic fields satisfy Maxwell's equations $\partial E_{y} / \partial y=4 \pi e\left(Z n_{\mathrm{i}}-n_{\mathrm{e}}\right)$ and $\partial B_{z} / \partial y=-4 \pi j_{x} / c \approx-4 \pi e n_{\mathrm{e}} v_{\mathrm{d} x} / c$. Integrating these two equations in the region 

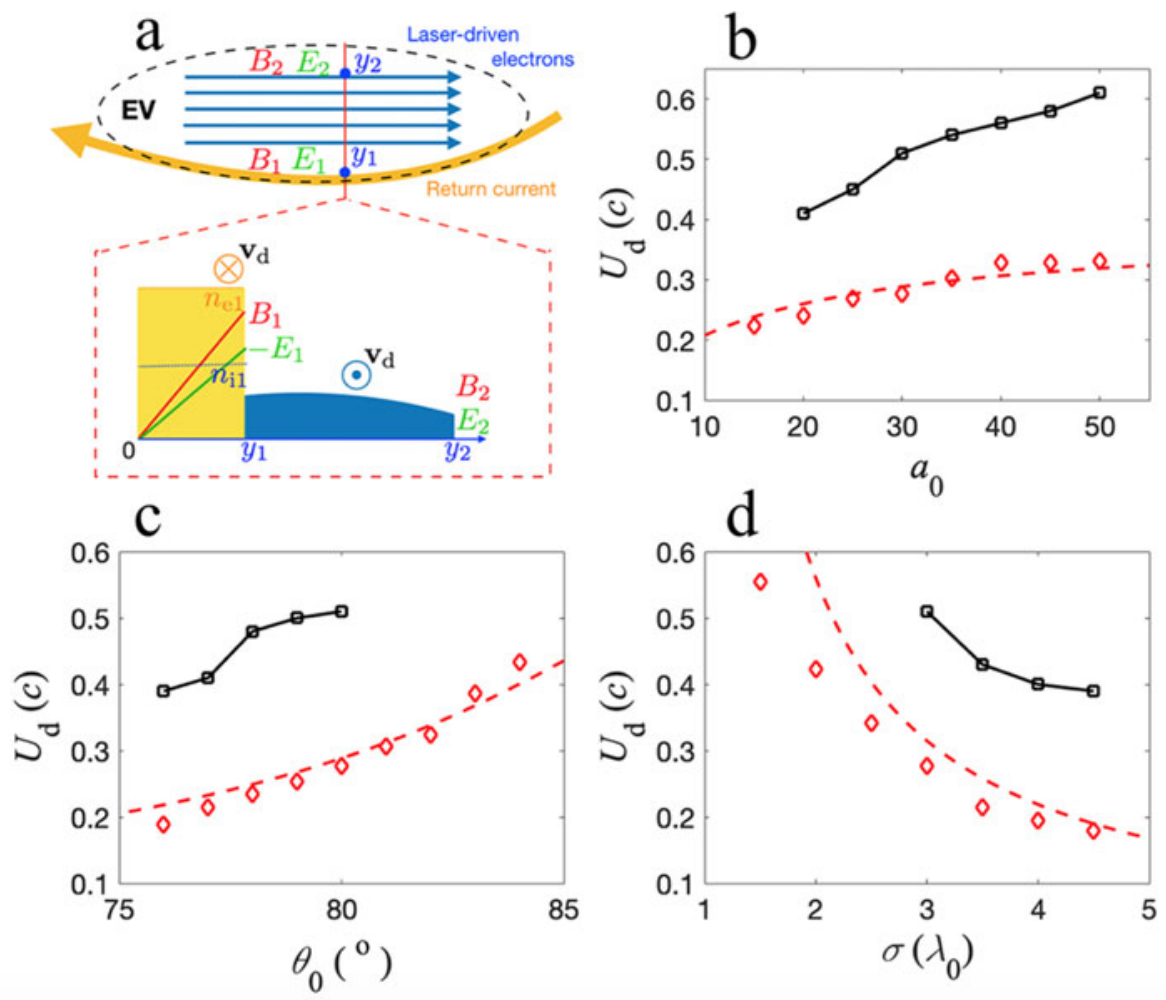

FIGURE 4. Analytical model to calculate the EV drift velocity. (a) The one-dimensional EV model with two oppositely travelling electron streams. The drift velocity of EV and the maximum velocity of the accelerated protons (black squares) are plotted as functions of $(b) a_{0},(c) \theta_{0}$ and $(d) \sigma$, respectively. These parameters are set to their default values $a_{0}=30, \theta_{0}=80^{\circ}$ and $\sigma=3 \lambda_{0}$, when not being scanned. The red diamonds and dashed lines in $(b-d)$ represent the drift velocities in PIC simulations and the fit suggested by (3.2).

of laser-induced current gives an estimate for the drift velocity of the EV

$$
U_{\mathrm{d}}=\frac{\int_{y_{1}}^{y_{2}} v_{\mathrm{d}} n_{\mathrm{e}} \mathrm{d} y}{\int_{y_{1}}^{y_{2}} n_{\mathrm{e}} \mathrm{d} y} \approx\left(\frac{E_{1}}{B_{1}}+\frac{4 \pi e N_{\mathrm{i}}}{B_{1}}\right)^{-1} c,
$$

where $N_{\mathrm{i}}=Z n_{\mathrm{i} 1} \sigma\left(1-\exp \left[\left(y_{1}-y_{2}\right) / \sigma\right]\right)$ is the ion areal density inside the EV. Thus, the drift velocity of an EV depends on $N_{i}$ as well as the electric and magnetic fields at $y_{1}$.

The first term in the bracket on the right-hand side of (3.1) can be obtained by integrating Maxwell's equations in the return current layer (note that the $\boldsymbol{E} \times \boldsymbol{B}$ drift is the dominating term in this region, as illustrated in figure $3(b)$, so that $v_{\mathrm{d}}$ can be estimated by $c E_{y} / B_{z}$ ), which gives $E_{1} / B_{1}=-\sqrt{1-Z n_{\mathrm{i} 1} / n_{\mathrm{e} 1}}$. From this it follows that $\left|E_{1} / B_{1}\right|$ varies over a range of $\sim 0.1$, that is typically an order of magnitude smaller than the variation of the second term in (3.1). As a representative value, we assume $Z n_{\mathrm{i} 1} \approx n_{\mathrm{e} 1} / 2$, yielding $E_{1} / B_{1} \approx-0.7$. 
An estimate of the second term in (3.1) relies on the fact that the amplitude of $B_{1}$ in the EV approximately equals the magnetic field in the laser created channel (see figure 1). Consider the case where all the electrons in the channel move forward with velocity $\sim c$, and the bottom of the channel is at the position $y=y_{1}$. Then $B_{1} \approx 4 \pi e Z n_{\mathrm{i} 1} \sigma\left[1-\exp \left(-R_{\mathrm{ch}} / \sigma\right)\right]$, where $R_{\mathrm{ch}}=\lambda_{0} \sqrt{\gamma_{a} n_{\mathrm{c}} / 4 \pi^{2} n_{\mathrm{m}}}$ is the characteristic radius of the laser-driven channel (Borisov et al. 1995; Pukhov, Sheng \& Meyer-ter Vehn 1999), and $n_{\mathrm{m}}$ is the local plasma density corresponding to the maximum laser penetration depth in the NCD plasma.

From Snell's law, we obtain $\eta \sin \alpha=\eta_{0} \sin \theta_{0}$, where $\eta \approx 1-n_{\mathrm{e}} /\left(2 \gamma_{a}\right)$ is the refractive index of the plasma [the initial value is $\eta_{0} \approx 1-n_{0} /\left(2 \gamma_{a}\right)$, and $\alpha$ is the angle between the laser propagation direction and $y$ axis. Thus, by setting $\alpha=\pi / 2$, the density corresponding to the maximum penetration of the laser is obtained as $n_{\mathrm{m}} / n_{\mathrm{c}}=2 \gamma_{a}-\left(2 \gamma_{a}-n_{0} / n_{\mathrm{c}}\right) \sin \theta_{0}$.

For large $\mathrm{EVs}\left(N_{\mathrm{i}} \approx Z n_{\mathrm{i} 1} \sigma\right)$, the drift velocity in terms of laser-plasma parameters can then be approximated as

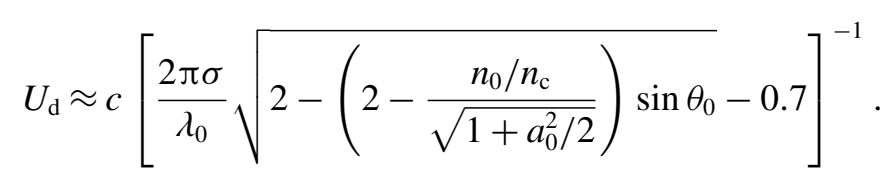

The most important prediction of (3.2) is that the drift velocity, and so the maximum proton energy, does not depend crucially on the laser intensity, since the plasma density at the incident point is typically $n_{0} / n_{\mathrm{c}} \ll a_{0}$. Therefore, controlling the laser incident angle and the plasma density gradient can be effectively used to adjust the proton energy. This agrees well with the PIC simulations as shown by figure $4(b-d), U_{\mathrm{d}}$ depends strongly on the laser incident angle and plasma scale length, and weakly on the laser intensity (the scaling flattens further as $n_{0}$ decreases).

The maximum proton speed obtained in the PIC simulations are overlaid on the plot in figure $4(b-d)$ with black squares, and they are approximately twice the corresponding drift velocity. There are cases where no accelerated proton energy is plotted above $U_{\mathrm{d}}$. This indicates that protons cannot be captured by the EV for those parameters, either because the acceleration field $E_{x}$ is too weak (in figure $4(b)$ when $a_{0}=10$ ) or because the $\mathrm{EV}$ is drifting too fast (for the cases when $U_{\mathrm{d}}>0.4 c$ ). In this case one could increase the values of $a_{0}, \sigma$, or reduce the incidence angle $\theta_{0}$ as suggested in figure $4(b-d)$.

Unlike most other proton acceleration mechanisms, which require high laser intensity to reach high ion energies, the scheme proposed here provides accessible degrees of freedom to control the acceleration process, allowing accelerating protons to hundreds of $\mathrm{MeV}$ with a narrow energy spread. According to figure 4 the laser parameters required to produce a 100-200 MeV monoenergetic, self-injected proton beam are within reach of existing laser facilities.

\section{Discussion}

The grazing incidence of the laser pulse allows the laser to accelerate hot electrons parallel to the target surface, the energy in the resulting coherent motion can subsequently be transferred to protons during the EV-acceleration stage. If the incident angle is too small, the EV will be depleted at a premature state of the proton acceleration.

Also, when the incident angle of the laser is less than some critical angle (so that the plasma density on both sides of the laser becomes significantly higher than in the electron depletion region created by the laser in the middle), the laser self-channelling 

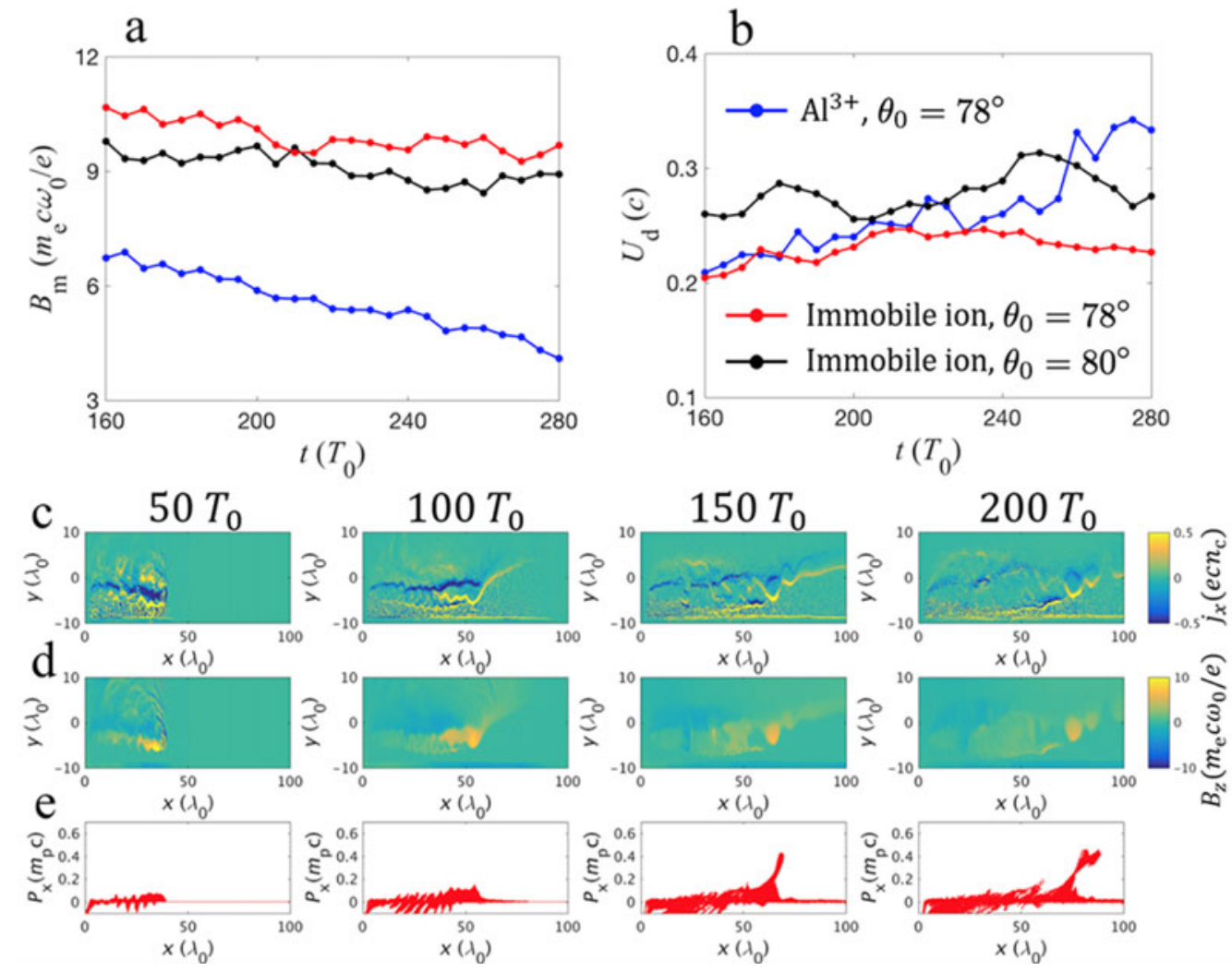

FIGURE 5. The effect of dynamical background heavy ions. Time evolution of $(a)$ the maximum magnetic field and $(b)$ the EV drift velocity. The blue line corresponds to a simulation with mobile $\mathrm{Al}^{3}+$ background ions and a laser incidence angle of $78^{\circ}$. The red and black lines show cases with immobile ions, and angles $78^{\circ}$ and $80^{\circ}$, respectively. Snapshots of $(c)$ the current density $j_{x},(d)$ the out-of-plane magnetic field $B_{z}$ and $(e)$ the $x-P_{x}$ proton phase space at $t=50 T_{0}, 100 T_{0}, 150 T_{0}$ and $200 T_{0}$, respectively, in a simulation with a mobile background heavy ion species, $\mathrm{Al}^{3+}$.

effect becomes important, and the laser-driven electrons will be trapped in the channel running towards the high-density region. In this situation no EV forms. Therefore, we only consider the grazing incident angle, above $75^{\circ}$, in this work.

It should be noted that the immobile ion background assumption used in the simulation of figure 1 is reasonable, as the required low charge-to-mass ratio (i.e. low degree of ionisation) of the heavy ions can be well satisfied in the region where the EV propagates and the ions are being accelerated. The high intensity laser light that can ionise the plasma to a higher degree never reaches this region, as it is fully depleted by the time the vortex is fully formed. To illustrate the effect of dynamical background heavy ions, here we present a simulation where we replace the immobile ions with $\mathrm{Al}^{3+}$. The initial ratio of $\mathrm{Al}^{3+}$ and $\mathrm{H}^{+}$in the expanding near-critical-density plasma is assumed to be 18:1. In addition, the laser incident angle is $\theta_{0}=78^{\circ}$ (as motivated below), all other parameters are unchanged from those in figure 1 .

As seen in figure 5, the EV is slowly dissipated as ions are scattered out of the vortex by its electrostatic field. As a result, the EV loses energy and its lifetime is reduced. Figure 5(a) shows that the maximum magnetic field in the EV is reduced by 

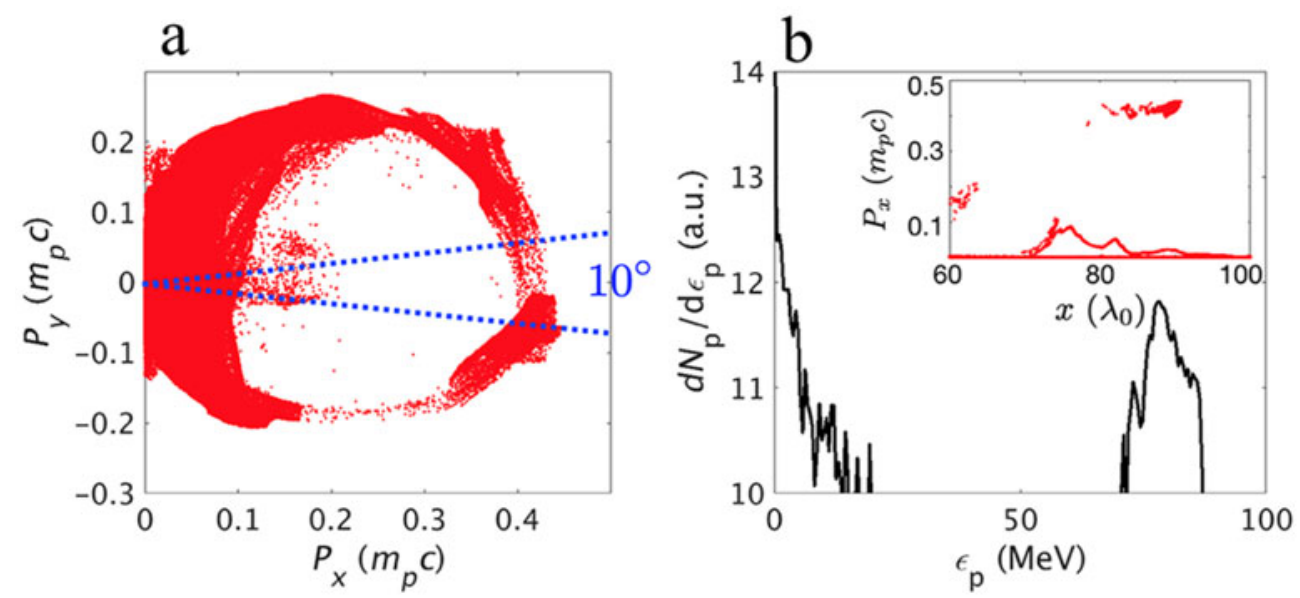

FIgURE 6. Accelerated protons in a simulation with mobile ions. (a) Proton distribution in the $P_{x}-P_{y}$ phase space, and $(b)$ proton energy spectrum within an opening angle of $10^{\circ}$ at $t=200 T_{0}$. The inset in $(b)$ shows the $x-P_{x}$ phase space map of these protons.

$40 \%$ within $120 T_{0}$ in the mobile background ion case $\left(\mathrm{Al}^{3+}\right)$, while in the immobile ion case, the reduction is only approximately $10 \%$. The decrease in magnetic field results in a slight increase in the EV drift velocity as shown in figure $5(b)$. This can be understood from (3.1), $U_{\mathrm{d}} \sim B_{1} / N_{\mathrm{i}} \sim \sigma \sqrt{n_{\mathrm{m}} / \gamma_{a}}$ and $B_{1} \sim \sqrt{\gamma_{a} n_{\mathrm{m}}}$, which yield $U_{\mathrm{d}} \sim$ $\gamma_{a} / \sigma B_{1}$. Because of this, there is no analogous spectral broadening effect as in shock wave acceleration (Macchi, Nindrayog \& Pegoraro 2012).

As shown in figure 5(e), the qualitative features of the process and the overall dynamics remain the same with mobile ions. Importantly, the fields of the EV remain sufficiently strong during the time scale needed for proton acceleration $\left(\sim 50 T_{0}\right)$. To compensate for the somewhat weaker fields in the EV, the incident angle of the laser pulse has been reduced. To ensure proton self-injection, the maximum incident angle is $\theta_{0}=78^{\circ}$, which leads to an acceleration energy of $\sim 80 \mathrm{MeV}$. This is approximately $60 \%$ of the value obtained in the immobile ion case. Otherwise, the proton phase space and energy spectrum are similar to those with immobile ions, as shown in figure 6. These results suggest that heavier background ion species (that tend to have smaller charge-to-mass ratio due to partial ionisation) are preferable for achieving a more energetic proton beam with longer duration.

Finally, we present a three-dimensional (3-D) simulation of the considered set-up. We use a circularly polarised laser with a reduced peak intensity $I_{0}=3 \times 10^{20} \mathrm{~W} \mathrm{~cm}^{-2}$ and an elliptical focal spot $\left(I=I_{0} \exp \left(-y^{2} / w_{y}^{2}-z^{2} / w_{z}^{2}\right)\right.$, where $w_{y}=3 \lambda_{0}$ and $\left.w_{z}=9 \lambda_{0}\right)$. The initial plasma density profile is assumed to be uniform in the $z$ direction, the laser incident angle $\theta_{0}=75^{\circ}$, and other parameters are the same as quoted for the 2 -D simulation of figure 1 . The resolution needed to be reduced compared to two dimensions, for computational feasibility: the simulation box is $x \times y \times z=100 \lambda_{0} \times$ $20 \lambda_{0} \times 40 \lambda_{0}$, which is sampled by $1000 \times 200 \times 400$ cells with 3 macro particles per cell for both electrons and ions. Circular polarisation is chosen as it can generate a higher current density, leading to a stronger EV. The linearly polarised laser is known to produce fast electron 'jets', twice per laser cycle, which appears as an unwanted loss mechanism. An elliptical laser focus spot is employed to elongate the flux rope, 

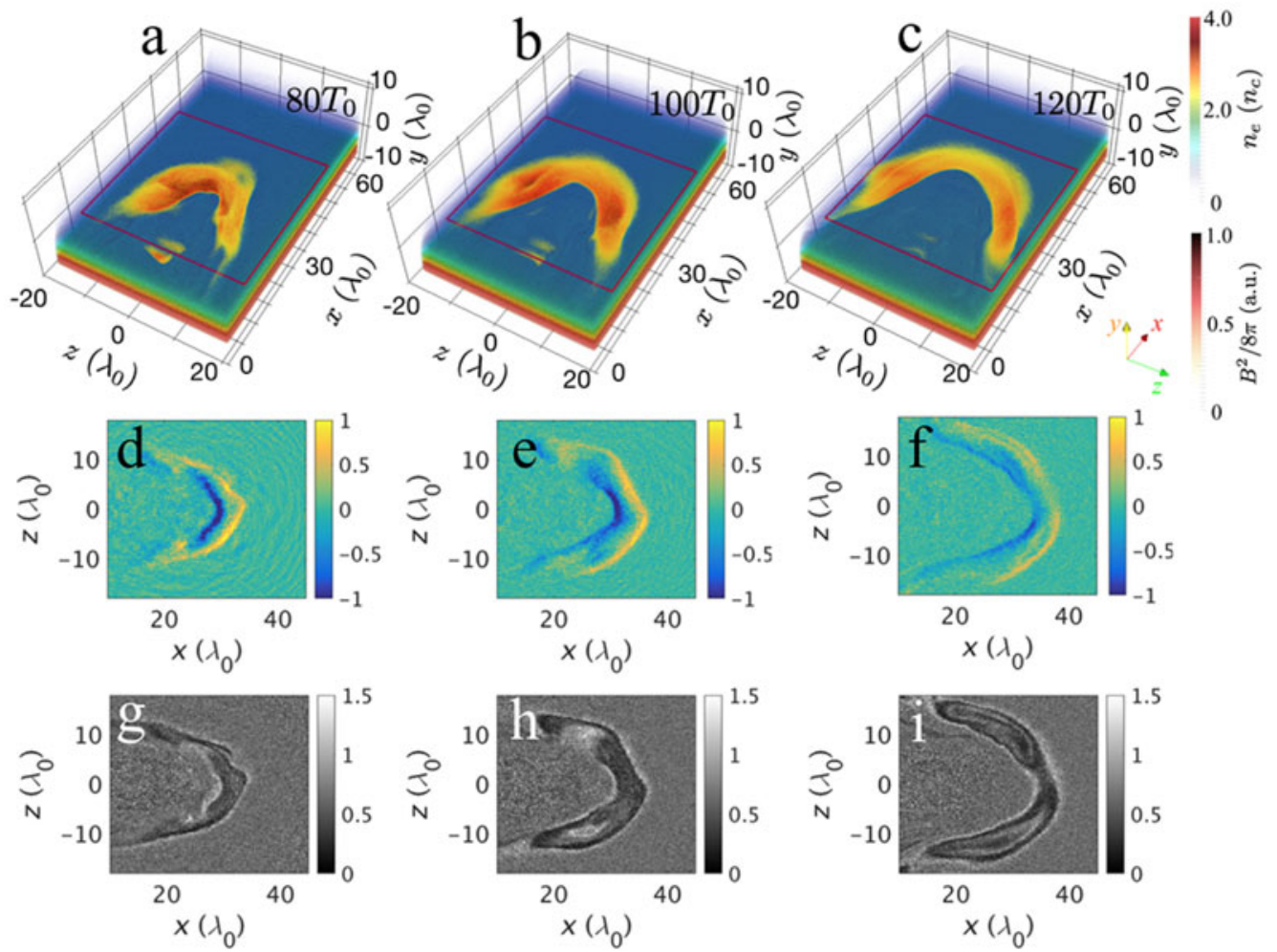

Figure 7. The 3-D structure of the electron vortex. Magnetic energy density (white-orange colour scale) and the electron density (rainbow-colour scale) in the 3-D PIC simulation at $(a) t=80 T_{0},(b) 100 T_{0}$ and $(c) 120 T_{0} ;(d-f)$ and $(g-i)$ show the $x$ component of the electric field, and the electron density depletion for the cross-section at $y=-1.6 \lambda_{0}$ (for regions marked by the red rectangles in $a-c$ ).

thereby mitigating the leakage of electrons along the field lines, and a lower laser incident angle is used to ensure proton injection.

Figure 7 illustrates that the electron vortex has a 'magnetic flux rope' structure in 3-D space, where the magnetic field is highly concentrated in a flux tube that lays in the $x-z$ plane and resembles a horseshoe. The plasma density depletion and charge separation fields are similar to those in the 2-D simulations, and the structure stays stable for more than $40 T_{0}$. The formation and drifting processes of the EV in 2-D and 3-D simulations are essentially the same, which indicates the robustness of the underlying physics, i.e. the electrons trapped in the vortex are subject to an $\boldsymbol{E} \times \boldsymbol{B}$ drift which drives the collective drift motion of the vortex structure; the strong magnetic pressure leads to charge separation in the vortex and a population of protons along its path are captured and accelerated (see figure 8).

However, in three dimensions the electrons can escape along the magnetic field lines in the third dimension ('leaking' effect), leading to a reduction of the current and the magnetic energy density. As a result, the lifetime of the electron vortex is expected to be shorter, usually resulting in a lower energy of the accelerated protons. As shown in figure $8(a)$, a monotonically decreasing proton spectrum is produced, with a cutoff energy of approximately $30 \mathrm{MeV}$, and the proton number (above $10 \mathrm{MeV}$ ) per solid angle is estimated to be $10^{4} / \mathrm{MeV} / \mathrm{msr}$. The lack of the high energy peak is caused 

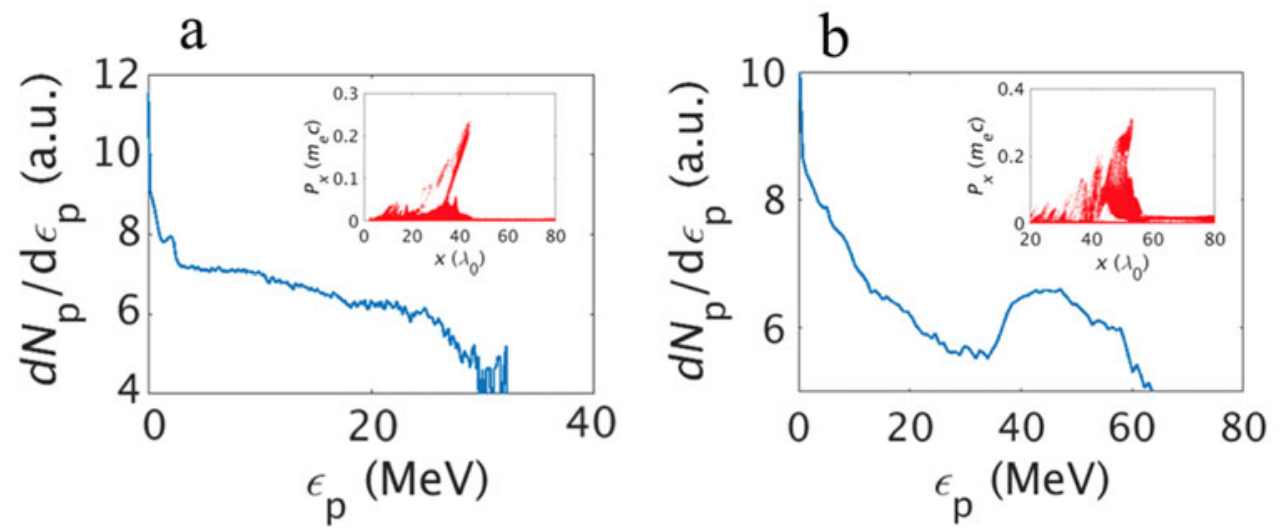

FIGURE 8. Accelerated proton spectra in 3-D simulations within opening angle $\left|\theta_{y}\right|=$ $\arctan \left(\left|P_{y}\right| / P_{x}\right)<10^{\circ}$ at simulation time $t=140 T_{0}$. (a) Laser peak intensity $I_{0}=3 \times$ $10^{20} \mathrm{~W} \mathrm{~cm}{ }^{-2}$, with elliptical focus spot $w_{y}=3 \lambda_{0}$ and $w_{z}=9 \lambda_{0}$, incident at $\theta_{0}=75^{\circ} .(b)$ 'Quasi-2-D' case, with $w_{z} \gg w_{y}$, laser peak intensity $I_{0}=5.5 \times 10^{20} \mathrm{~W} \mathrm{~cm}^{-2}$, incident at $\theta_{0}=78^{\circ}$.

by the rapid decay of the EV due to electron leakage along field lines. That the EV loses energy, and its drift velocity changes as it rises towards the lower-density region, happens within the time scale of the proton acceleration, resulting in a different spectral shape and low proton energies. The unwanted effects of the leakage can be mitigated by increasing the intensity and the ellipticity of the laser spot shape. As an illustration, in figure $8(b)$ we show a case with an extremely elongated laser spot, $w_{z} \gg w_{y}$ [i.e. $I=I_{0} \exp \left(-y^{2} / w_{y}^{2}\right)$ ], where a laser of intensity $I_{0}=5.5 \times 10^{20} \mathrm{~W} \mathrm{~cm}^{-2}$ is incident at $\theta_{0}=78^{\circ}$, recovering the high energy proton peak in the spectrum. A quantitative study on the differences between 2-D and 3-D cases, induced by hot electron leakage, is computationally very expensive; the detailed optimisation of the presented scheme in three dimensions is left for future studies.

\section{Conclusions}

In conclusion, we show that when a laser pulse irradiates an NCD plasma in a grazing angle, in the presence of a sharp, pre-formed target-normal density gradient, the resulting intense electric currents form an electron vortex after the laser depletion. The magnetic pressure leads to a significant reduction in the electron density in the centre of the vortex, and the electric field created by the charge separation can accelerate ions. The drift velocity of the EV can be effectively tuned by the incident angle of the laser and the density gradient of the plasma. Using PIC simulations we demonstrate that protons initially at rest can be captured and accelerated to maximum twice of the EV drift velocity with a narrow energy spread.

\section{Acknowledgements}

The authors acknowledge fruitful discussions with S. Newton, J. Martins, E. Siminos, J. Ferri, I. Thiele and the rest of the PLIONA team. This work is supported by the Knut and Alice Wallenberg Foundation, the European Research Council (ERC-2014-CoG grant 647121), the National Natural Science Foundation of China (grant no. 11505262), the Ministry of Science and Technology of the People's 
Republic of China (grant nos. 2016YFA0401102 and 2018YFA0404803) and the Strategic Priority Research Program of the Chinese Academy of Sciences (grant no. XDB16). Simulations were performed on resources at Chalmers Centre for Computational Science and Engineering (C3SE) provided by the Swedish National Infrastructure for Computing (SNIC).

\section{Supplementary movie}

Supplementary movie is available at https://doi.org/10.1017/S0022377819000485.

\section{REFERENCES}

Albertazzi, B., D’Humières, E., Lancia, L., Dervieux, V., Antici, P., Böcker, J., Bonlie, J., BReil, J., Cauble, B., Chen, S. N. et al. 2015 A compact broadband ion beam focusing device based on laser-driven megagauss thermoelectric magnetic fields. Rev. Sci. Instrum. 86 (4), 043502.

Angus, J. R., Richardson, A. S., Ottinger, P. F., Swanekamp, S. B. \& Schumer, J. W. 2014 Nonquasineutral electron vortices in nonuniform plasmas. Phys. Plasmas 21 (11), 112306.

Arber, T. D., Bennett, K., Brady, C. S., Lawrence-Douglas, A., Ramsay, M. G., Sircombe, N. J., Gillies, P., Evans, R. G., Schmitz, H., Bell, A. R. et al. 2015 Contemporary particle-in-cell approach to laser-plasma modelling. Plasma Phys. Control. Fusion 57 (11), 1-26.

Bin, J. H., Ma, W. J., Wang, H. Y., Streeter, M. J. V., Kreuzer, C., Kiefer, D., Yeung, M., Cousens, S., Foster, P. S., DromeY, B. et al. 2015 Ion acceleration using relativistic pulse shaping in near-critical-density plasmas. Phys. Rev. Lett. 115, 064801.

Borghesi, M. \& MACCHI, A. 2016 Laser-Driven Ion Accelerators: State of the Art and Applications. pp. 221-247. Springer.

Borisov, A. B., Shiryaev, O. B., McPherson, A., Boyer, K. \& Rhodes, C. K. 1995 Stability analysis of relativistic and charge-displacement self-channelling of intense laser pulses in underdense plasmas. Plasma Phys. Control. Fusion 37 (5), 569-597.

Bulanov, S. S., Brantov, A., Bychenkov, V. Y., ChVykov, V., Kalinchenko, G., Matsuoka, T., Rousseau, P., Reed, S., Yanovsky, V., Krushelnick, K. et al. 2008 Accelerating protons to therapeutic energies with ultraintense, ultraclean, and ultrashort laser pulses. Med. Phys. 35 (5), 1770-1776.

Bulanov, S. S., Bychenkov, V. Y., ChVykov, V., Kalinchenko, G., LitzenberG, D. W., Matsuoka, T., Thomas, A. G. R., Willingale, L., Yanovsky, V., Krushelnick, K. et al. 2010 Generation of gev protons from $1 \mathrm{PW}$ laser interaction with near critical density targets. Phys. Plasmas 17 (4), 043105.

Bulanov, S. V. \& Esirkepov, T. Z. 2007 Comment on 'collimated multi-MeV ion beams from high-intensity laser interactions with underdense plasma'. Phys. Rev. Lett. 98, 049503.

Bulanov, S. V., Lontano, M., Esirkepov, T. Z., Pegoraro, F. \& Pukhov, A. M. 1996 Electron vortices produced by ultraintense laser pulses. Phys. Rev. Lett. 76, 3562-3565.

Daido, H., Nishiuchi, M. \& PirozhKov, A. S. 2012 Review of laser-driven ion sources and their applications. Rep. Prog. Phys. 75 (5), 056401.

Fiuza, F., Fonseca, R. A., Tonge, J., Mori, W. B. \& Silva, L. O. 2012 Weibel-instabilitymediated collisionless shocks in the laboratory with ultraintense lasers. Phys. Rev. Lett. 108, 235004.

Fuchs, J., Antici, P., D’humières, E., Lefebvre, E., Borghesi, M., Brambrink, E., Cecchetti, C., Kaluza, M., Malka, V., Manclossi, M. et al. 2006 Laser-driven proton scaling laws and new paths towards energy increase. Nat. Phys. 2, 48-54.

Gauthier, M., Lévy, A., D’Humières, E., Glesser, M., Albertazzi, B., Beaucourt, C., Breil, J., Chen, S. N., Dervieux, V., Feugeas, J. L. et al. 2014 Investigation of longitudinal proton acceleration in exploded targets irradiated by intense short-pulse laser. Phys. Plasmas 21 (1), 013102. 
Gordeev, A. V. \& Levchenko, S. V. 1998 Is the Abrikosov model applicable for describing electronic vortices in plasmas? J. Expl. Theor. Phys. Lett. 67 (7), 482-488.

Haberberger, D., Tochitsky, S., Fiuza, F., Gong, C., Fonseca, R. A., Silva, L. O., Mori, W. B. \& Joshi, C. 2012 Collisionless shocks in laser-produced plasma generate monoenergetic high-energy proton beams. Nat. Phys. 8 (1), 95-99.

Higginson, A., Gray, R. J., King, M., Dance, R. J., Williamson, S. D. R., Butler, N. M. H., Wilson, R., Capdessus, R., Armstrong, C., Green, J. S. et al. 2018 Near-100 MeV protons via a laser-driven transparency-enhanced hybrid acceleration scheme. Nat. Commun. 9, 724.

Hilz, P., Ostermayr, T. M., Huebl, A., Bagnoud, V., Borm, B., Bussmann, M., Gallei, M., Gebhard, J., Haffa, D., Hartmann, J. et al. 2018 Isolated proton bunch acceleration by a petawatt laser pulse. Nat. Commun. 9, 423.

Khudik, V., Yi, S. A., Siemon, C. \& Shvets, G. 2014 The analytic model of a laser-accelerated plasma target and its stability. Phys. Plasmas 21 (1), 013110.

Lemos, N., Martins, J. L., Dias, J. M., Marsh, K. A., Pak, A. \& Joshi, C. 2012 Forward directed ion acceleration in a lwfa with ionization-induced injection. J. Plasma Phys. 78 (4), 327-331.

LiU, B., Meyer-ter Vehn, J., Bamberg, K.-U., Ma, W. J., LiU, J., He, X. T., Yan, X. Q. \& Ruhl, H. 2016 Ion wave breaking acceleration. Phys. Rev. Accel. Beams 19, 073401.

Liu, B., Wang, H. Y., LiU, J., Fu, L. B., Xu, Y. J., Yan, X. Q. \& He, X. T. 2013 Generating overcritical dense relativistic electron beams via self-matching resonance acceleration. Phys. Rev. Lett. 110, 045002.

Ma, W. J., Kim, I. J., Yu, J. Q., Choi, I. W., Singh, P. K., Lee, H. W., Sung, J. H., Lee, S. K., Lin, C., LiAO, Q. et al. 2019 Laser acceleration of highly energetic carbon ions using a double-layer target composed of slightly underdense plasma and ultrathin foil. Phys. Rev. Lett. 122, 014803.

MACCHI, A. 2017 A review of laser-plasma ion acceleration. arXiv:1712.06443.

Macchi, A., Borghesi, M. \& PASsoni, M. 2013 Ion acceleration by superintense laser-plasma interaction. Rev. Mod. Phys. 85, 751.

Macchi, A., Nindrayog, A. S. \& Pegoraro, F. 2012 Solitary versus shock wave acceleration in laser-plasma interactions. Phys. Rev. E 85, 046402.

Mora, P. 2003 Plasma expansion into a vacuum. Phys. Rev. Lett. 90, 185002.

Nakamura, T., Bulanov, S. V., Esirkepov, T. Z. \& Kando, M. 2010 High-energy ions from near-critical density plasmas via magnetic vortex acceleration. Phys. Rev. Lett. 105, 135002.

NAKAmuRA, T. \& Mima, K. 2008 Magnetic-dipole vortex generation by propagation of ultraintense and ultrashort laser pulses in moderate-density plasmas. Phys. Rev. Lett. 100, 205006.

Naumova, N. M., Koga, J., Nakajima, K., Tajima, T., Esirkepov, T. Z., Bulanov, S. V. \& Pegoraro, F. 2001 Polarization, hosing and long time evolution of relativistic laser pulses. Phys. Plasmas 8 (9), 4149-4155.

NyCAnder, J. \& PAVlenko, V. P. 1991 Stationary propagating magnetic electron vortices. Phys. Fluids B: Plasma Phys. 3 (6), 1386-1391.

Pak, A., Kerr, S., Lemos, N., Link, A., Patel, P., Albert, F., Divol, L., Pollock, B. B., Haberberger, D., Froula, D. et al. 2018 Collisionless shock acceleration of narrow energy spread ion beams from mixed species plasmas using $1 \mu \mathrm{m}$ lasers. Phys. Rev. Accel. Beams 21, 103401.

Palmer, C. A. J., Schreiber, J., Nagel, S. R., Dover, N. P., Bellei, C., Beg, F. N., Bott, S., Clarke, R. J., Dangor, A. E., Hassan, S. M. et al. 2012 Rayleigh-Taylor instability of an ultrathin foil accelerated by the radiation pressure of an intense laser. Phys. Rev. Lett. 108, 225002 .

Pegoraro, F. \& Bulanov, S. V. 2007 Photon bubbles and ion acceleration in a plasma dominated by the radiation pressure of an electromagnetic pulse. Phys. Rev. Lett. 99, 065002.

Priest, E. R., Hornig, G. \& Pontin, D. I. 2003 On the nature of three-dimensional magnetic reconnection. J. Geophys. Res. 108 (A7), doi:10.1029/2002JA009812. 
Pukhov, A. 2001 Three-dimensional simulations of ion acceleration from a foil irradiated by a short-pulse laser. Phys. Rev. Lett. 86, 3562-3565.

Pukhov, A., Sheng, Z.-M. \& Meyer-Ter Vehn, J. 1999 Particle acceleration in relativistic laser channels. Phys. Plasmas 6 (7), 2847-2854.

Pukhov, A. \& MeYer-Ter Vehn, J. 1996 Relativistic magnetic self-channeling of light in nearcritical plasma: three-dimensional particle-in-cell simulation. Phys. Rev. Lett. 76, 3975-3978.

Richardson, A. S., Angus, J. R., Swanekamp, S. B., Ottinger, P. F. \& Schumer, J. W. 2013 Theory and simulations of electron vortices generated by magnetic pushing. Phys. Plasmas 20 (8), 082115.

Roth, M., Blazevic, A., Geissel, M., Schlegel, T., Cowan, T. E., Allen, M., Gauthier, J.-C., Audebert, P., Fuchs, J., Meyer-ter Vehn, J. et al. 2002 Energetic ions generated by laser pulses: a detailed study on target properties. Phys. Rev. ST Accel. Beams 5, 061301.

Rust, D. M. \& Kumar, A. 1996 Evidence for helically kinked magnetic flux ropes in solar eruptions. Astrophys. J. 464 (2), L199-L202.

Schreiber, J., Bolton, P. R. \& PARodi, K. 2016 Invited review article: 'Hands-on' laser-driven ion acceleration: a primer for laser-driven source development and potential applications. Rev. Sci. Instrum. 87 (7), 071101.

Silva, L. O., Marti, M., Davies, J. R., Fonseca, R. A., Ren, C., Tsung, F. S. \& Mori, W. B. 2004 Proton shock acceleration in laser-plasma interactions. Phys. Rev. Lett. 92, 015002.

Sylla, F., Flacco, A., Kahaly, S., Veltcheva, M., Lifschitz, A., Malka, V., D’Humières, E., ANDRIYASh, I. \& TIKHONCHUK, V. 2013 Short intense laser pulse collapse in near-critical plasma. Phys. Rev. Lett. 110, 085001.

Thaury, C., Quéré, F., Geindre, J.-P., Levy, A., Ceccotti, T., Monot, P., Bougeard, M., RÉau, F., D’Oliveira, P., Audebert, P. et al. 2007 Plasma mirrors for ultrahigh-intensity optics. Nat. Phys. 3, 424-429.

Toncian, T., Borghesi, M., Fuchs, J., D’Humières, E., Antici, P., Audebert, P., Brambrink, E., Cecchetti, C. A., Pipahl, A., Romagnani, L. et al. 2006 Ultrafast laser-driven microlens to focus and energy-select mega-electron volt protons. Science 312 (5772), 410-413.

Wiegelmann, T. \& BÜChneR, J. 2001 Evolution of magnetic helicity in the course of kinetic magnetic reconnection. Nonlinear Process. Geophys. 8 (3), 127-140.

Wilks, S. C., Langdon, A. B., Cowan, T. E., Roth, M., Singh, M., Hatchett, S., Key, M. H., Pennington, D., Mackinnon, A. \& Snavely, R. A. 2001 Energetic proton generation in ultra-intense laser-solid interactions. Phys. Plasmas 8 (2), 542.

YADAV, S. K., DAS, A. \& KAW, P. 2008 Propagation of electron magnetohydrodynamic structures in a two-dimensional inhomogeneous plasma. Phys. Plasmas 15 (6), 062308.

Yamada, M., Kulsrud, R. \& Ji, H. 2010 Magnetic reconnection. Rev. Mod. Phys. 82, 603-664.

YI, L. Q., Shen, B. F., PUKhov, A. \& FÜlÖP, T. 2018 Relativistic magnetic reconnection driven by a laser interacting with a micro-scale plasma slab. Nat. Commun. 9, 1601.

Zhai, S. H., Shen, B. F., Borghesi, M., Wang, W. P., Zhang, H., Kar, S., Ahmed, H., Li, J. F., LI, S. S., ZhAng, H. et al. 2019 Proton array focused by a laser-irradiated mesh. Appl. Phys. Lett. 114 (1), 013509. 